Research in Astronomy and Astrophysics manuscript no.

(LATEX: manscript ${ }^{\circ} 0172 . R 1 . t e x ;$ printed on July 27, 2021; 0:37)

\title{
Systematic Analysis of Low/Hard State RXTE Spectra of GX 339-4 to Constrain the Geometry of the System
}

\author{
Kalyani Bagri ${ }^{1}$, Ranjeev Misra ${ }^{2}$, Anjali Rao ${ }^{2}$, J. S. Yadav ${ }^{3}$ and S. K. Pandey ${ }^{1}$ \\ 1 Pt. Ravishankar Shukla University, Raipur, India; kalyanibagri@ gmail.com \\ 2 Inter University Center for Astronomy and Astrophysics, Pune \\ 3 Tata Institute of Fundamental Research, Mumbai
}

\begin{abstract}
One of the popular models for the low/hard state of Black Hole Binaries is that the standard accretion disk is truncated and the hot inner region produces via Comptonization, the hard X-ray flux. This is supported by the value of the high energy photon index, which is often found to be small $\sim 1.7(<2)$ implying that the hot medium is seed photons starved. On the other hand, the suggestive presence of a broad relativistic Fe line during the hard state would suggest that the accretion disk is not truncated but extends all the way to the inner most stable circle orbit. In such a case, it is a puzzle why the hot medium would remain photon starved. The broad Fe line should be accompanied by a broad smeared reflection hump at $\sim 30 \mathrm{keV}$ and it may be that this additional component makes the spectrum hard and the intrinsic photon index is larger, i.e. $>2$. This would mean that the medium is not photon deficient, reconciling the presence of a broad Fe line in the observed hard state. To test this hypothesis, we have analyzed the RXTE observations of GX 339-4 from the four outbursts during 2002-2011 and identify the observations when the system was in the hard state and showed a broad Fe line. We have then attempted to fit these observations with models, which include smeared reflection to understand whether the intrinsic photon index can indeed be large. We find that, while for some observations the inclusion of reflection does increase the photon index, there are hard state observations with broad Fe line that have photon indices less than 2 .
\end{abstract}

Key words: accretion, accretion disks - stars: black holes - X-rays: binaries - X-rays: individual (GX 339-4)

\section{INTRODUCTION}

Black hole binaries are some of the highly variable astronomical objects observed in the X-ray sky and their variability is revealed in both spectral and timing characteristics. The occasional presence of Quasi 
QPOs etc. are some of the manifestations of variability observed in timing properties. The variability in spectral behavior can be described in terms of spectral states exhibiting different shapes, which can be interpreted as being due to varying relative contribution from two or more spectral components. The two major components observed in the spectrum include a multi-temperature thermal component (Mitsuda et al. (1984); Shakura \& Sunyaev (1973)) originating from an accretion disk and a power law tail due to inverse Comptonized radiation from the corona. The thermal component is mainly present towards softer X-ray bands $(\leq 10 \mathrm{keV})$ and it dominates the overall spectrum when a system is in the soft spectral states. The copious amount of soft seed photons originating from the disk results in a steep spectrum with a photon index $\Gamma \geq 2$. However, the strength of the thermal emission from the disk weakens during the hard spectral state and the spectrum is dominated by a hard power law component that extends towards the softer energies as well. The weak thermal component implies a smaller number of soft seed photons resulting in a relatively flat spectrum with a photon index of $\Gamma<2$. The transient black hole binaries undergo a series of spectral state transitions during an outburst in a systematic manner, which is often depicted as Q-shaped track in hardness-intensity diagrams (Fender et al., 2009). These diagrams have shown that a transient black hole binary is observed in the hard state when it enters the outburst, followed by transition to the hard intermediate state (HIMS), soft intermediate state (SIMS) and soft state (Belloni et al., 2011; Fender et al., 2009; Homan \& Belloni, 2005). The order is reversed as the outburst begins to decline and a system may show a few excursions to different spectral states (see Belloni et al., 2011; Fender et al., 2009; Homan \& Belloni, 2005). However, the overall scheme of state transitions remains the same for transient black hole binaries. On the other hand, the persistent black hole binaries have not shown a predictable trend of state transitions as in the case of transient black hole binaries, for example, Cyg X-1 remains in the hard state most of the time with occasional excursions to the soft state (Grinberg et al., 2013).

The seed photon starved hard spectral state is generally understood in terms of an accretion disk truncated far from the central black hole and the inner region being replaced by the hot inner flow which is sometimes modeled as an Advection Dominated Accretion Flow (ADAF; Narayan \& Yi, 1995). The truncation of disk may be described by the disk evaporation model, which was first proposed by Meyer et al. (2000) and later extended by Liu et al. (2002) and Mever-Hofmeister \& Mever (2003). The model considers a continuous evaporation of material from the disk, which feeds the corona. Oiao \& Liu (2009) calculated the evaporation rate as a function of disk radius and showed that it is maximum at a certain radius for a given value of the viscosity parameter. The model predicts that the truncation of disk will occur at a radius where the accretion rate is equal to the maximum evaporation rate and the inner disk will survive the evaporation only when the accretion rate is higher than the maximum evaporation rate. The model successfully explains the finding of a truncated accretion disk (e.g. Plant et al., 2014, 2015; Tomsick et al., 2009, etc.) as a consequence of the low mass accretion rate during the hard state, and an extended disk approaching the Innermost Stable Circular Orbit (ISCO) owing to the higher mass accretion rate in the soft state. It also provides a framework to the models addressing the steady jet emission during the hard states (see Belloni (2010) and Done et al. (2007) for detailed reviews).

The estimation of the inner disk radius using the observational data is crucial to understanding the 
the accretion disk is truncated during the hard states, the Fe emission line should be narrow and symmetric as the relativistic effects due to gravitational field of the black hole are weaker on the disk material. However, in soft states, the line should be broadened and skewed by the strong general relativistic effects in the inner region. Therefore, finding a broad $\mathrm{Fe}$ line in hard state serves as a strong evidence towards the extension of the disk in the inner regions and hence the violation of the truncated disk model. In such a scenario, a seed photon starved system during the hard state remains a puzzle. In several reports, authors have not only found a broad and skewed Fe line in the hard state, but also estimated the spin parameter by modeling the line profile and found a value of spin parameter consistent with the results obtained from the study of continuum and reflection component. Miniutti et al. (2004) studied the three BeppoSAX observations of a black hole candidate XTE J1650-500 during its 2001-02 outburst and perhaps detected the source in the power law dominated hard state $(\Gamma \sim 1.8)$ in one of the observations. They find a broad and strongly relativistic Fe emission line in the spectrum. The modeling of the line revealed the presence of the disk extending to $\sim 1.34 R_{g}$, which led them to suggest the presence of a Kerr black hole in the system. The extension of the accretion disk to the inner region in another black hole binary Swift J1753.5-0127 is shown by Miller et al. (2006a) during the decline of its 2005 outburst, wherein the inner disk was found at or close to the ISCO by modeling the continuum with a number of models. Their results show that the disk can be present in the inner regions during the hard state at very low luminosities down to $L_{X} \simeq 0.003 L_{E d d}$. The same set of observations belonging to the hard state were further studied by Reis et al. (2009a) to find the spin parameter and inclination angle using a model that included a power law and reflection component. The innermost emitting region was shown to extend close to $\sim 3.1 R_{g}$ and a spin parameter of $\sim 0.76$ was reported by assuming that the inner edge of the disk is at the ISCO. Another result presenting the violation of the truncated disk model is shown in Reis et al. (2009b) wherein the authors extracted the spectrum of XTE J1118+480 in its hard state using Chandra and RXTE and found the presence of thermal component with disk temperature of $\sim 0.21 \mathrm{keV}$, which suggested that the emission is originating from an accretion disk extending close to the radius of marginal stability. XTE J1817-330 was studied by Rykoff et al. (2007) using Swift observations during its 2006 outburst. They used the disk continuum model and found the inner disk radius to be consistent with the ISCO. They showed that the luminosity follows the relation $L_{X} \propto T^{4}$ roughly during the decline of the outburst, which led them to suggest the presence of a geometrically stable disk in the inner regions at accretion rate as low as $0.001 L_{E d d}$. Reis et al. (2010) studied eight black hole binaries in their hard spectral state. The modeling of the disk continuum revealed that the luminosity in all the systems is consistent with the relation $L_{X} \propto T^{4}$ down to $\sim 5 \times 10^{-4} L_{E d d}$. The six sources showed truncation radius not larger than $10 R_{g}$ and the Fe line detected in four of the black hole binaries at luminosities down to $1.5 \times 10^{-3} L_{E d d}$ excluded a truncated disk. Other studies on the finding of the inner disk radius close to the ISCO during low/hard state include Reynolds et al. (2010) and Reynolds \& Miller (2013). Hence, there are good number of evidence suggesting that the disk may not be truncated in the hard spectral state.

While several results show the violation of the truncation model, there are also number of results that support the truncation of the disk in the hard state. Tomsick et al. (2009) have reported the detection of 
and RXTE observations. The truncated disk scenario is supported by their results wherein the inner disk radius is shown to increase by a factor of $>27$ as compared to the value found when the source was bright. Although, the inner disk radius is shown to be dependent on the inclination angle (i.e. $R_{i n}>35 R_{g}$ at $i=0^{\circ}$ and $R_{i n}>175 R_{g}$ at $i=30^{\circ}$ ), the results provide a direct evidence for the absence of the inner disk at low luminosities. The detection of Fe line in the hard state of GX 339-4 is reported by Shidatsu et al. (2011) and an inner disk radius of $\sim 13.3 R_{g}$ is estimated by modeling its profile. Their results indicate that the accretion disk evolves inward as luminosity increases in the range $\sim 0.001<L_{X} / L_{E d d}<\sim 0.02$ when the source remains in the hard state. The hints of disk recession can be found in Petrucci et al. (2014), wherein the spectral results of five Suzaku observations taken during the decline of 2010-2011 outburst are presented. An inner disk radius of $<10-30 R_{g}$ is found in the first two observations, however it remains unconstrained for the latter observations due to low statistics. Plant et al. (2014) have presented a very detailed study of reflection component observed in the joint spectral fitting of PCA and HEXTE spectra for the three outbursts of GX 339-4. Their results support the truncation of the inner disk during the hard state, and a decrease in the coronal height in the soft state. Truncation of disk in GX 339-4 in the hard state is again corroborated by Plant et al. (2015) with the spectral studies performed using XMM-Newton and Suzaku observations. Kolehmainen et al. (2014) studied GX 339-4 with XMM-Newton data in its hard state, and used both Fe line emission and disc continuum methods to measure the inner radius of the accretion disk. They estimated the effects of instrumental and modeling uncertainties and showed that both the methods provide results consistent with the truncated disc model. In addition, there are several reports where authors reanalyzed the results suggesting violation of truncation of disk and reclaimed the finding of truncated disk. A reanalysis of BeppoSAX observation of GX 339-4 by Done \& Gierliński (2006) confirmed the finding of a broad Fe line caused by the extreme relativistic effects previously reported by Miller et al. (2002) and Miniutti et al. (2004). However, the authors reinstated the truncation of the inner disk by showing that the relativistic smearing can be significantly reduced by considering resonance Fe $\mathrm{K}$ line absorption from an outflowing disk wind. Done \& Diaz Trigo (2010) reanalyzed XMM-Newton data of GX 339-4 studied by Miller et al. (2006b) and Reis et al. (2008) which claimed the detection of broad Fe line in the hard state. A detailed reanalysis showed that MOS data of XMM-Newton is heavily piled-up and a broad Fe line is an artifact of the same. The spectrum extracted with PN timing mode data of the same observation revealed a narrow line consistent with the truncation of disk in hard state. Other reports showing the truncation of accretion disk in the hard state for various black hole binaries include Basak \& Zdziarski (2016), Rao Jassal \& Vadawale (2015), Yuan \& Narayan (2014), Cabanac et al. (2009), and Gierliński et al. (2008).

The Fe line emission is just one feature of the reflection component detected in black hole binaries. Another major feature of this component is the broad hump that appear at $\sim 10-30 \mathrm{keV}$. The reflection hump appearing towards higher energies, if not modeled properly, can give rise to an artificial hardening to an intrinsically soft spectrum. Therefore, it may be possible that reflection can be the reason behind hardening of an originally soft spectrum. It implies that the system is not seed photon starved and hence the finding of a broad Fe line can also be reconciled. In order to test the hypothesis, we have studied the black hole binary GX 339-4, which is already an object in the debate of truncation of accretion disk in hard state (e.g. 
black hole with a mass of $5.8 \pm 0.5 M_{\circ}$ (Hynes et al., 2003) and distance of $>6 \mathrm{kpc}$ (Hynes et al., 2004). GX 339-4 has shown multiple outbursts in the past, which have been regularly monitored with RXTE. We have studied 4 outbursts of the object during 2002-2011 and in particularly its spectra. Our motivation is to fit the spectra with and without reflection component, in order to understand the effect the component has on the photon index. In the next section we will discuss the observations and the results found in the present work are discussed in the sections 2 and 3 respectively.

\section{OBSERVATIONS AND SPECTRAL FITTING}

This work presents a spectral analysis of pointed observations of the black hole binary GX 339-4 with Proportional Counter Array (PCA; Jahoda et al., 1996) onboard Rossi X-ray Timing Explorer (RXTE) during 2002-2011. We have studied a total of 1160 pointed observations available on the High Energy Astrophysics Science Analysis Archive (HEASARC) covering the four outbursts (2002-03, 2004-05, 200607, 2010-11) of the transient object. We study Standard-2 spectra from PCA data, and the spectral fitting was performed in the energy range of 3-20 keV using the spectral fitting package XSPEC version 12.8.2. All spectral parameters are presented with a confidence interval of $90 \%$ unless otherwise mentioned. The spectra studied here belong to the different spectral states of the four outbursts, which is manifested by their variable shapes. The spectral components have varying relative strengths, indicating the changing geometry and evolution of the physical processes in the system. Therefore, the spectra require different spectral models and we follow a scheme of spectral fitting where we begin with the simplest model of a power law absorbed by the interstellar medium and increase the complexity of the model by including the thermal emission from the accretion disk, $\mathrm{Fe} \mathrm{K} \alpha$ emission and reflection continuum. All the models are listed in the Table (1) and a description of the fitting procedure is given below.

We begin the spectral fitting of all the spectra with the simplest model of an absorbed power law listed as M1 in the Table (1) and mention the number of spectra explained with the model with alphabets A, B, C and D belonging to 2002-03, 2004-05, 2006-07 and 2010-11 outbursts respectively. The interstellar absorption is modeled with the model wabs (Morrison \& McCammon, 1983) available in XSPEC and the absorption column is fixed at $3.74 \times 10^{21} \mathrm{~cm}^{2}$. It is found that a total of $261([\mathrm{~A}] 66 ;[\mathrm{B}] 82 ;[\mathrm{C}] 96,[\mathrm{D}] 17)$ spectra provide a good fit $\left(\Delta \chi^{2}<1.2\right)$ with this model. Since there is no soft disk component seen in this class of spectra, it is expected that the observations providing a good fit with this model belong to the hard spectral state. Fig (1) shows the variation of flux as a function of time and it can be seen that all the spectra falling in this class appear towards the lower flux values shown in red.

It is clear that the remaining spectra which are not well explained with the model M1 include other spectral components and the spectral model needs to be modified to account for the additional components. Therefore, the remaining spectra are fitted with the canonical model of a black hole binary consisting of a soft thermal emission from a disk and a non-thermal component originating from the corona. The disk component is modeled with a multicolor disk black body model DISKBB (Mitsuda et al., 1984) and the non-thermal component is modeled with POWERLAW. It is found that none of the remaining spectra are explained with the model and exhibit a larger residual close to $6 \mathrm{keV}$. Therefore, we include GAUSSIAN in 
model WABS * (DISKBB+POWERLAW+GAUSS IAN) is listed as M2 in Table (1). The width of Gaussian is allowed as a free parameter. A total of 301 ([A]61; [B]81; [C]38, [D]121) spectra are explained with this model giving reduced $\Delta \chi^{2} \leq 1.2$. As a next step in the spectral analysis, we allowed both the centroid energy and the width of Gaussian as free parameters and we name the model as M3 in Table (1). The model resulted in 210([A]31; [B]89; [C]52; [D]48) spectra giving reduced $\Delta \chi^{2} \leq 1.2$. Fig (1) shows the flux values for this model with pink color. It was found that some of the remaining spectra showed improvement in the spectral fitting when a narrow Gaussian line was included in the model in addition to the broad Gaussian. This resulted in a model M4 WABS* (DISKBB+POWERLAW+GAUSSIAN+GAUSSIAN). The model provides a good fit to 200 ([A]51; [B]52; [C]51, [D]46) spectra. For the spectral fitting of remaining spectra, the column density is allowed as a free parameter and this model M5 provides a good fit to 47 ([A]16; [B]5; $[C] 2 ;[D] 24)$ spectra. The remaining spectra are not explained with any of the above mentioned models resulting in higher $\Delta \chi^{2}$ values. Model systematic errors were introduced for these spectra and systematic errors of $0.5 \%, 1 \%, 2 \%$ and $3 \%$ allow to obtain a good fit for the $87([\mathrm{~A}] 25$; [B]4; [C]28, [D]30), 33 ([A]3; [B]5; [C]14, [D]11), 15 ([C]10, [D]5 and 6([B]1; [C]4;[D]1) spectra with model M5. Table (1) summarizes the list of models, free parameters, systematic error and the number of spectra fitted. There are a number of free parameters in the spectral models and we focused on the photon index and width of Fe line, in particular, for this study as discussed in the next section.

Table 1: List of the models, free parameters and the number of spectra providing the good fit.

\begin{tabular}{|c|c|c|c|}
\hline \multicolumn{2}{|l|}{ Model } & \multirow{2}{*}{$\begin{array}{l}\text { Free Parameters } \\
\Gamma, \mathrm{N}_{p l}\end{array}$} & \multirow{2}{*}{$\frac{\text { No.of spectra }}{261}$} \\
\hline$M_{1}$ & wabs*powerlaw & & \\
\hline$M_{2}$ & wabs(diskbb+powerlaw+gaussian) & $\mathrm{T}_{i n}, \mathrm{~N}_{d b b}, \Gamma, \mathrm{N}_{p l}, \sigma, \mathrm{N}_{g a u}$ & 301 \\
\hline$M_{3}$ & wabs(diskbb+powerlaw+gaussian) & $\mathrm{T}_{i n}, \mathrm{~N}_{d b b}, \Gamma, \mathrm{N}_{p l}, \mathrm{E}_{g a u}, \sigma, \mathrm{N}_{g a u}$ & 210 \\
\hline$M_{4}$ & wabs(diskbb+gaussian+powerlaw+gaussian) & $\mathrm{T}_{i n}, \mathrm{~N}_{d b b}, \Gamma, \mathrm{N}_{p l}, \mathrm{~N}_{\text {gau } 1}, \mathrm{E}_{\text {gau } 2}, \sigma_{2}, \mathrm{~N}_{\text {gau } 2}$ & 200 \\
\hline \multirow[t]{5}{*}{$M_{5}$} & wabs(diskbb+gaussian+powerlaw+gaussian) & $\mathrm{T}_{i n}, \mathrm{~N}_{d b b}, \Gamma, \mathrm{N}_{\text {pl }}, \mathrm{E}_{\text {gau } 1}, \mathrm{~N}_{\text {gau } 1}, \mathrm{E}_{\text {gau } 2}, \sigma_{2}, \mathrm{~N}_{\text {gau } 2}, N_{H}$ & 47 \\
\hline & $0.5 \% \mathrm{SE}$ & & 87 \\
\hline & $1.0 \% \mathrm{SE}$ & & 33 \\
\hline & $2.0 \% \mathrm{SE}$ & & 15 \\
\hline & $3.0 \% \mathrm{SE}$ & & 6 \\
\hline
\end{tabular}

\section{Notes.}

$\mathrm{T}_{i n}$ and $\mathrm{N}_{d b b}$ are the temperature at the inner disk radius and normalization of D ISKBB.

$\Gamma$ and $\mathrm{N}_{p l}$ are photon index and normalization of POWERLAW. $\mathrm{E}_{g a u}, \sigma$ and $\mathrm{N}_{g a u}$ are centroid energy, FWHM and normalization of GAUSSIAN. The subscript ' 1 ' and '2' are used with parameters of the narrow and broad GAUSSIAN respectively in models M4 and M5.

SE stands for model systematic uncertainty.

\section{RESULTS AND DISCUSSION}

The scheme of spectral fitting discussed above is expected to separate the hard spectra from the softer ones. It is expected that the hard spectra would appear towards the lower flux values. This is justified by the flux 


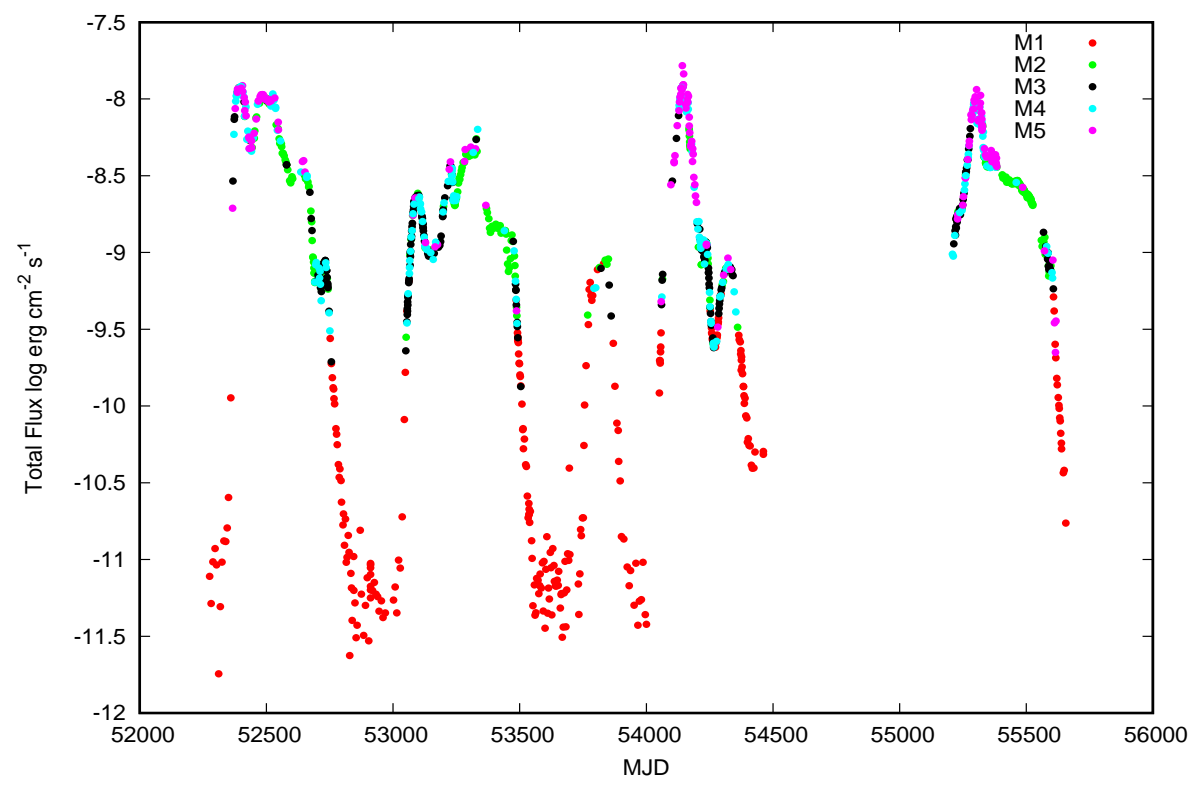

Fig. 1: The plot shows the total flux in 3-20 keV energy range as a function of time for the four outbursts during 2002-11. The spectra providing good fit with model M1, M2, M3, M4 and, M5 are shown with red, green, black, cyan, and pink respectively.

seen that a majority of the spectra explained with the model M1 appear towards lower flux values, however, a few of the spectra appear towards higher flux as well. The spectra explained with model M2, M3, M4 and M5 are shown in green, black, cyan, and pink respectively. It is noticeable that the spectra explained with model M4 and M5 appear towards higher flux in the plot.

The standard model of accretion disk describe the thermal emission from the disk in terms of blackbody radiation emitted by the annuli of different radii, integrated over the inner and outer edge of the disk. The inner edge of the disk may extend down to the ISCO at the maximum beyond which no stable orbits are allowed. The model, however does not account for the non-thermal emission commonly seen in the spectrum of black hole binaries. The overall spectrum of black hole binaries including thermal and non-thermal components is explained in terms of a geometrically thin and optically thick accretion disk along with geometrically thick and optically thin hot inner flow (Narayan \& Yi, 1995). The hot inner flow intercepts a fraction of soft seed photons from the accretion disk which is inverse Compton scattered. The photons escaping directly from the hot flow give rise to the non-thermal component in the spectrum. A fraction of Comptonized radiation is reflected by the accretion disk resulting in an additional characteristic spectrum. The reflection spectrum mainly consists of a broad continuum called Compton hump at $\sim 30 \mathrm{keV}$ and an Fe $\mathrm{K} \alpha$ line at $6.4 \mathrm{keV}$.

In the soft spectral state, the accretion rate is high and the disk extends in the inner regions approaching the ISCO. The effects of general and special relativity are prominent on the emission from the inner region resulting in a broad and skewed $\mathrm{Fe}$ line. On the other hand, the accretion disk is expected to be truncated far away from the black hole during the hard spectral state, where the effects of relativity are weaker and a narrow Fe line is expected to be observed. Therefore, the presence of a broad and skewed Fe line during hard state presents a violation of the truncated disk scenario. The disk extending close to the ISCO during hard 


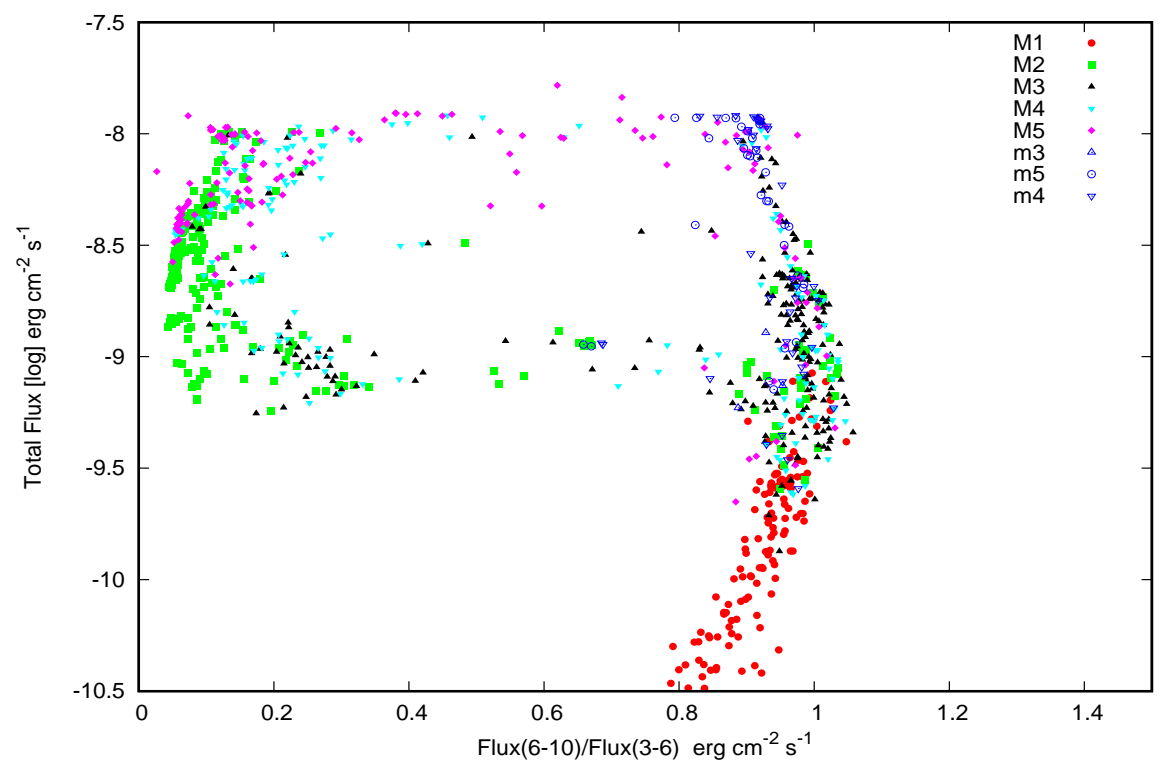

Fig. 2: Hardness Intensity Diagram for the four outbursts during 2002-11. The spectra providing good fit with model M1, M2, M3, M4 and, M5 are shown with red, green, black, cyan, and pink respectively. The spectra with $\Gamma<1.8$ and $\sigma>1.5 \mathrm{keV}$ are shown with blue.

belonging to the hard state with broader Fe line need to be investigated in detail in order to understand the discrepancy. We study the Hardness-Intensity diagram for the four outbursts during 2002-11. For the sake of clarity, we represent the spectra providing good fit with model M1, M2, M3, M4 and M5 are shown with red, green, black, cyan, and pink respectively in Fig 2 . While the spectra with $\Gamma<1.8$ and $\sigma>1.5 \mathrm{keV}$ are shown by blue color with different symbols. These spectra are fitted with models (M3, M4 and M5) are indicated as $\mathrm{m} 3, \mathrm{~m} 4$ and $\mathrm{m} 5$ in Fig 2 . The hard state spectrum is defined as a thermal Comptonization component with photon index $\Gamma \sim 1.8$, a weak disk emission and a moderate reflection component. We selected the spectra with photon index $<1.8$ and broad Fe line with FWHM $>1.5 \mathrm{keV}$ and found a total of 76 [A:20; B:19; C:21; D:16] spectra matching these criteria. If we interpret the broad Fe line as a result of the relativistic effects in the vicinity of the black hole, these spectra present a case where the truncation of the disk stands violated. Therefore, we focus our further investigation mainly on this set of spectra. Fig (3), which is the same plot as Fig (11) shows all the 76 cases marked as blue points with almost all of them appearing towards higher flux values. The evolution of Fe line width and photon index with time is shown in Fig (4) for the first outburst. The two occurrences of spectra with $\Gamma<1.8$ and $\sigma>1.5 \mathrm{keV}$ can be seen in Fig (4).

The higher value of soft energy flux and the finding of broad Fe line will be consistent if these spectra belong to soft spectral state with $\Gamma>1.8$. Therefore, a hypothesis is proposed that the observed spectra with broad Fe line actually belong to the soft state, however the hardening of spectra may artificially be resulted by the reflection hump that appear towards the higher energies. Therefore, we have added reflection component reflionx to the 76 spectra from the four outbursts. The change in the power law index is studied before and after adding the reflection component in the model. We found that the error bars of photon index are either very large or zero for the 40 spectra. So we have provided the spectra for the 36 observations 


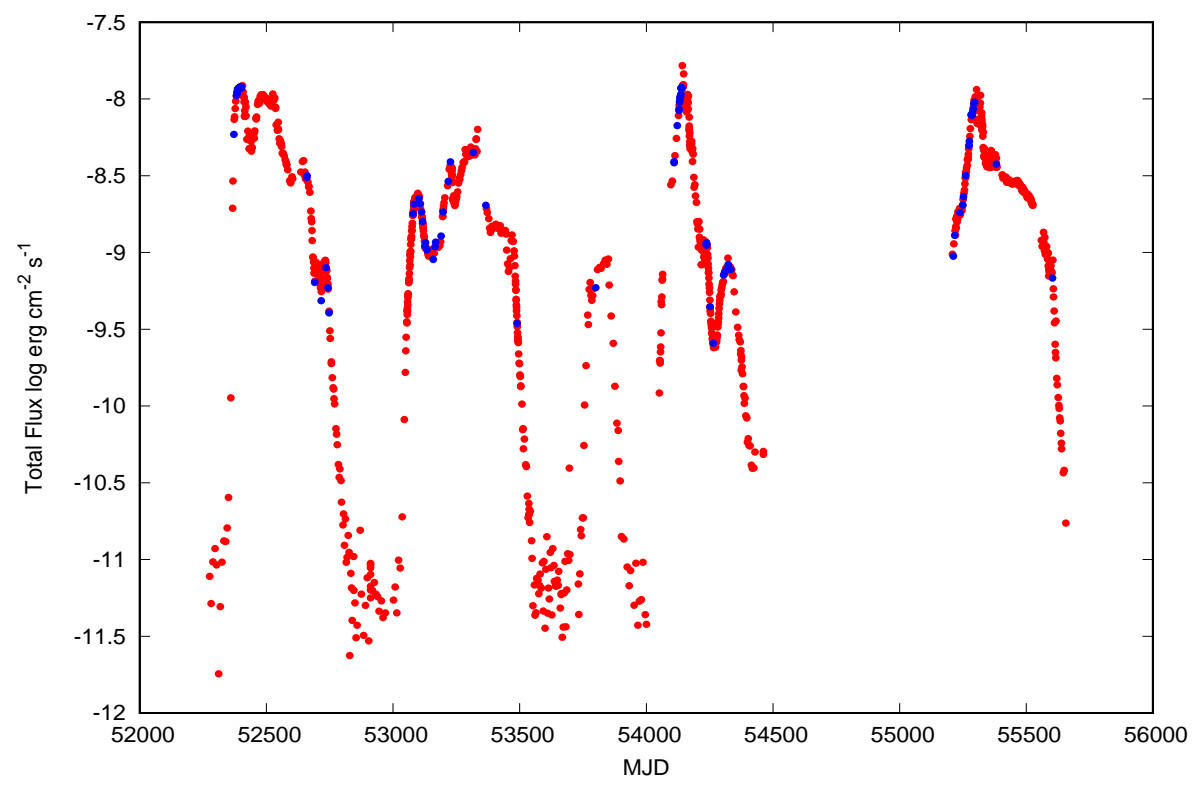

Fig. 3: Total flux in 3-20 keV energy range as a function of time for the four outbursts during 2002-11. The observations with $\Gamma<1.8$ and $\sigma>1.5 \mathrm{keV}$ are shown with blue points and remaining observations with red. It is noticeable that the blue points appear towards the higher flux values.

green line representing the same value of power law index before and after adding the reflection. The points lying above and below this line show the softening and hardening of spectra respectively. For some of the spectra, the photon index after adding reflection was found to be higher than the best-fit value obtained without reflection component. However, the photon index with reflection component was found to be $>2$ only for two spectra and the remaining spectra continued to provide photon index $<2$. Therefore, the occurrence of hard spectrum and broad Fe line is not understood in the light of contribution from the reflection component. One of the possible solution is to model the spectrum with two Comptonization models (see Yamada et al., 2013; ; Basak et al., 2017), where a soft Comptonization component is considered in addition to the main Comptonization component. The model used by Basak et al. (2017) to study the hard state spectrum of Cyg X-1 consists of reflection from both Comptonization components. The soft Comptonization component models the soft excess towards lower energies in their work. The model yields the best-fit with $R_{i n}=13-20 R_{g}$, indicating a truncated accretion disk in hard state.

\section{CONCLUSIONS}

The two main features of the reflection component observed in the spectrum of black hole binaries include an Fe emission line at $6.4 \mathrm{keV}$ and broad Compton hump at $\sim 30 \mathrm{keV}$. The reflection hump appearing towards higher energies can give rise to an artificial hardening to an intrinsically soft spectrum. In order to test the hypothesis, we studied spectra from the four outbursts of GX 339-4 using RXTE/PCA data observed between 2002 to 2011 . We studied the spectra with different models of increasing complexity. We particularly shortlisted those cases where a broad Fe line $(\sigma>1.5 \mathrm{keV})$ was observed in the hard state with $\Gamma<1.8$. These spectra are refitted by adding reflection component and the values of the photon index before and after adding the reflection were compared. It is found that the addition of reflection component in the 


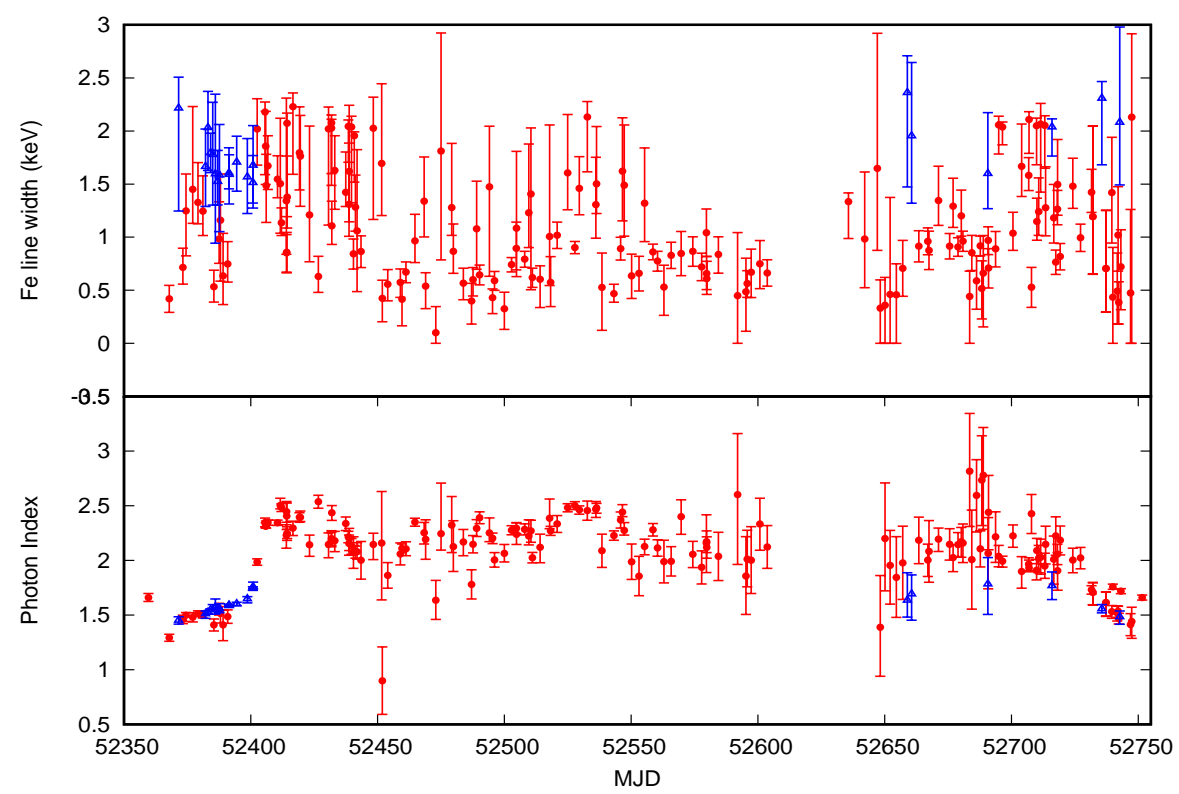

Fig. 4: The plot shows the evolution of Fe line width (top panel) and photon index (bottom panel) with time for 2002-03 outburst. The observations with $\Gamma<1.8$ and $\sigma>1.5 \mathrm{keV}$ are shown with blue points and remaining observations with red.

index $<1.8$ showed photon index $>2$ after addition of reflection component. However, there are several spectra where the photon index remains $\lesssim 1.8$. Therefore, the results show that the reflection component does not completely explain the puzzle about the existence of broad Fe line in the hard spectral state.

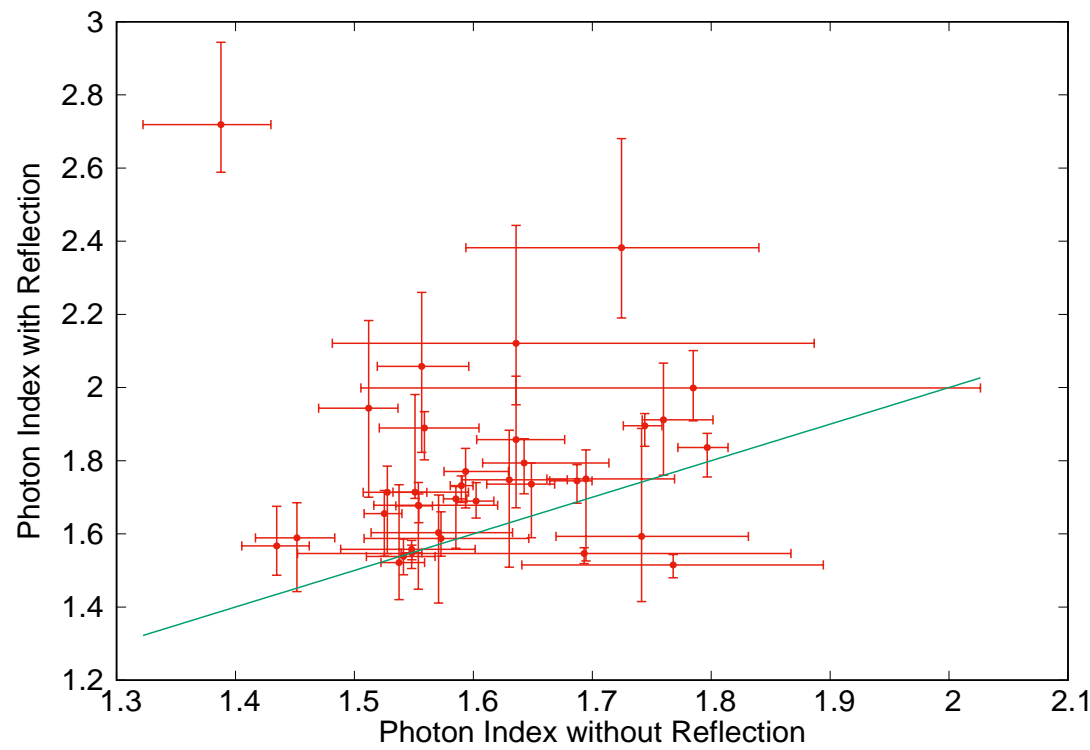

Fig. 5: The values of photon index with reflection versus the photon index without reflection for spectra with $\Gamma<1.8$ and $\sigma>1.5 \mathrm{keV}$ from all the four outbursts. The plot includes the values of photon indices from 36 spectra where the parameter is constrained before and after the addition of reflection component. 


\section{References}

Basak, R., \& Zdziarski, A. A. 2016, MNRAS, 458, 2199

Basak, R., Zdziarski, A. A., Parker, M., \& Islam, N. 2017, arXiv:1705.06638

Belloni, T. M. 2010, in Lecture Notes in Physics, Berlin Springer Verlag, Vol. 794, Lecture Notes in Physics, Berlin Springer Verlag, ed. T. Belloni, 53

Belloni, T. M., Motta, S. E., \& Muñoz-Darias, T. 2011, Bulletin of the Astronomical Society of India, 39, 409

Cabanac, C., Fender, R. P., Dunn, R. J. H., \& Körding, E. G. 2009, MNRAS, 396, 1415

Done, C., \& Diaz Trigo, M. 2010, MNRAS, 407, 2287

Done, C., \& Gierliński, M. 2006, MNRAS, 367, 659

Done, C., Gierliński, M., \& Kubota, A. 2007, A\&A Rev., 15, 1

Fender, R. P., Homan, J., \& Belloni, T. M. 2009, MNRAS, 396, 1370

Gierliński, M., Done, C., \& Page, K. 2008, MNRAS, 388, 753

Grinberg, V., Hell, N., Pottschmidt, K., et al. 2013, A\&A, 554, A88

Homan, J., \& Belloni, T. 2005, Ap\&SS, 300, 107

Hynes, R. I., Steeghs, D., Casares, J., Charles, P. A., \& O’Brien, K. 2003, ApJ, 583, L95

Hynes, R. I., Steeghs, D., Casares, J., Charles, P. A., \& O’Brien, K. 2004, ApJ, 609, 317

Jahoda, K., Swank, J. H., Giles, A. B., et al. 1996, in Proc. SPIE, Vol. 2808, EUV, X-Ray, and Gamma-Ray Instrumentation for Astronomy VII, ed. O. H. Siegmund \& M. A. Gummin, 59

Kolehmainen, M., Done, C., \& Díaz Trigo, M. 2014, MNRAS, 437, 316

Liu, B. F., Mineshige, S., Meyer, F., Meyer-Hofmeister, E., \& Kawaguchi, T. 2002, ApJ, 575, 117

Meyer, F., Liu, B. F., \& Meyer-Hofmeister, E. 2000, A\&A, 361, 175

Meyer-Hofmeister, E., \& Meyer, F. 2003, A\&A, 402, 1013

Miller, J. M., Homan, J., \& Miniutti, G. 2006a, ApJ, 652, L113

Miller, J. M., Homan, J., Steeghs, D., et al. 2006b, ApJ, 653, 525

Miller, J. M., Fabian, A. C., Wijnands, R., et al. 2002, ApJ, 570, L69

Miniutti, G., Fabian, A. C., \& Miller, J. M. 2004, MNRAS, 351, 466

Mitsuda, K., Inoue, H., Koyama, K., et al. 1984, PASJ, 36, 741

Morrison, R., \& McCammon, D. 1983, ApJ, 270, 119

Narayan, R., \& Yi, I. 1995, ApJ, 452, 710

Petrucci, P.-O., Cabanac, C., Corbel, S., Koerding, E., \& Fender, R. 2014, A\&A, 564, A37

Plant, D. S., Fender, R. P., Ponti, G., Muñoz-Darias, T., \& Coriat, M. 2014, MNRAS, 442, 1767

Plant, D. S., Fender, R. P., Ponti, G., Muñoz-Darias, T., \& Coriat, M. 2015, A\&A, 573, A120

Qiao, E., \& Liu, B. F. 2009, PASJ, 61, 403

Rao Jassal, A., \& Vadawale, S. V. 2015, Research in Astronomy and Astrophysics, 15, 45

Reis, R. C., Fabian, A. C., \& Miller, J. M. 2010, MNRAS, 402, 836

Reis, R. C., Fabian, A. C., Ross, R. R., \& Miller, J. M. 2009a, MNRAS, 395, 1257

Reis, R. C., Fabian, A. C., Ross, R. R., et al. 2008, MNRAS, 387, 1489

Reis, R. C., Miller, J. M., \& Fabian, A. C. 2009b, MNRAS, 395, L52 
Reynolds, M. T., \& Miller, J. M. 2013, ApJ, 769, 16

Reynolds, M. T., Miller, J. M., Homan, J., \& Miniutti, G. 2010, ApJ, 709, 358

Rykoff, E. S., Miller, J. M., Steeghs, D., \& Torres, M. A. P. 2007, ApJ, 666, 1129

Shakura, N. I., \& Sunyaev, R. A. 1973, A\&A, 24, 337

Shidatsu, M., Ueda, Y., Tazaki, F., et al. 2011, PASJ, 63, S785

Tomsick, J. A., Yamaoka, K., Corbel, S., et al. 2009, ApJ, 707, L87

Yamada, S., Makishima, K., Done, C., et al. 2013, PASJ, 65, 80

Yuan, F., \& Narayan, R. 2014, ARA\&A, 52, 529 